\title{
A design methodology for switched discrete time linear systems with applications to automotive roll dynamics control *
}

\author{
Selim Solmaz ${ }^{\mathrm{a}, *}$, Robert Shorten $^{\mathrm{a}}$, Kai Wulff $^{\mathrm{b}}$, Fiacre Ó Cairbre $^{\mathrm{c}}$

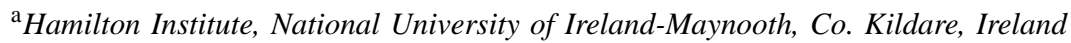 \\ ${ }^{\mathrm{b}}$ Technische Universität Berlin, Elektrotechnik und Informatik, Fachgebiet Regelungssysteme, D-10587 Berlin, Germany \\ ${ }^{\mathrm{c}}$ Department of Mathematics, National University of Ireland-Maynooth, Co. Kildare, Ireland
}

\begin{abstract}
In this paper we consider the asymptotic stability of a class of discrete-time switching linear systems, where each of the constituent subsystems is Schur stable. We first present an example to motivate our study, which illustrates that the bilinear transform does not preserve the stability of a class of switched linear systems. Consequently, continuous time stability results cannot be transformed to discrete time analogs using this transformation. We then present a subclass of discrete-time switching systems, that arise frequently in practical applications. We prove that global attractivity for this subclass can be obtained without requiring the existence of a common quadratic Lyapunov function (CQLF). Using this result we present a synthesis procedure to construct switching stabilizing controllers for an automotive control problem, which is related to the stabilization of a vehicle's roll dynamics subject to switches in the center of gravity (CG) height.
\end{abstract}

\section{Introduction}

Many control problems that arise in automotive engineering lead naturally to solutions that involve switching between a set of stabilizing controllers. Examples include speed control systems, and robust rollover mitigation systems [1]. In this paper we consider one such problem, where switching arises naturally due to changes in the vehicle parameters. Specifically, we consider the design of robust switched controllers that prevent instabilities due to abrupt changes in the center of gravity position.

Typically, switched linear controllers are designed using linear matrix inequalities (LMIs); see [1] for an example of such a design in the automotive roll dynamics control context. More often than not, LMI based control system design is based on quadratic Lyapunov functions, and is iterative in nature, requiring multiple searches before a controller satisfying certain performance criteria is found. Design methods that are constructive, in the manner of pole placement, say, for linear systems, are generally not available for the

\footnotetext{
* This paper was not presented at any IFAC meeting.

* Corresponding author. Tel: +353 1 7086100; Fax: +353 1 7086269.

Email addresses: selim.solmaz@nuim.ie (Selim Solmaz), robert.shorten@nuim.ie (Robert Shorten), wulffecontrol.tu-berlin.de (Kai Wulff),

Fiacre.oCairbre@maths.nuim.ie (Fiacre Ó Cairbre).
}

design of switched systems. One such method was however initially proposed in [2], and further developed in [3]. The basic problem addressed here is to study the discrete time analog of this system class. To show that this is not a trivial exercise we present the following example.

Example 1.1 Consider the following stable LTI systems,

$\Sigma_{A_{i}}: \quad \dot{x}=A_{i} x, \quad A_{i} \in \mathbb{R}^{3 \times 3}$,

with the constituent system matrices

$A_{1}=\left[\begin{array}{ccc}-19 & 0 & 0 \\ 0 & -9 & 0 \\ 0 & 0 & -0.25\end{array}\right], \quad A_{2}=\left[\begin{array}{ccc}-19 & 0 & 0 \\ -10 & -9 & 0 \\ -18.75 & 0 & -0.25\end{array}\right], \quad A_{3}=\left[\begin{array}{ccc}-19 & 0 & 18.75 \\ 0 & -9 & 8.75 \\ 0 & 0 & -0.25\end{array}\right]$

These three matrices all share the same eigenvalues, and they satisfy the conditions of Theorem 3.1 given in [2]. Therefore, one can conclude that the continuous time switched system $\Sigma_{A_{i}}$ above is stable. Now consider the bilinear mapping [4] below

$A_{d, i}=\left(A_{i}-I\right)^{-1}\left(A_{i}+I\right), \quad i \in\{1,2,3\}$,

where $I \in \mathbb{R}^{3 \times 3}$ is the identity matrix. The resulting discrete 
time matrices are

$A_{d, 1}=\left[\begin{array}{ccc}0.9 & 0 & 0 \\ 0 & 0.8 & 0 \\ 0 & 0 & -0.6\end{array}\right], \quad A_{d, 2}=\left[\begin{array}{ccc}0.9 & 0 & 0 \\ 0.1 & 0.8 & 0 \\ 1.5 & 0 & -0.6\end{array}\right], \quad A_{d, 3}=\left[\begin{array}{ccc}0.9 & 0 & -1.5 \\ 0 & 0.8 & -1.4 \\ 0 & 0 & -0.6\end{array}\right]$

It is sufficient to show that there exists a switching sequence between the matrices $\left\{A_{d, 1}, A_{d, 2}, A_{d, 3}\right\}$ such that the system

$\Sigma_{A_{d, i}}: x(k+1)=A(k) x(k) \quad$ for $\quad A(k) \in\left\{A_{d, 1}, A_{d, 2}, A_{d, 3}\right\}$,

is unstable. We simply consider the incremental switching sequence $A_{d, 3} \rightarrow A_{d, 2} \rightarrow A_{d, 1}$; then the dynamics of the system evolve according to the product

$$
A_{d}=A_{d, 1} A_{d, 2} A_{d, 3}
$$

Since the eigenvalues of $A_{d}$ are $\{0.512,-0.081,1.944\}$, then with one eigenvalue outside the unit circle, this switching sequence, repeated periodically, is unstable.

This example shows that, unlike LTI systems, the Bilinear transform does not necessarily preserve the stability of linear time-varying systems. This observation may have implications for control system design. Traditionally, the approach to relate continuous-time linear time invariant (LTI) Hurwitz stability results to discrete-time LTI Schur stability counterparts requires the use of the bilinear transform. Our example is consistent with the results reported in a recent paper [4], where it is noted that while quadratic Lyapunov functions are preserved under the Bilinear transform, other nonquadratic Lyapunov functions are not. Unfortunately, the example demonstrates that matters are much worse than reported in this paper; namely, that not only are non-quadratic functions not preserved under this mapping, but also stability need not be.

Fortunately, it is possible to modify the proof of Theorem 3.1 in [2] to place additional discrete time conditions on the system matrices to guarantee the global attractivity, and hence the exponential stability [5] of the origin for this system class.

\section{Stability of a class of discrete-time switched systems}

Consider the discrete time linear time-varying system

$x(k+1)=A(k) x(k)$,

where $x(k) \in \mathbb{R}^{n}$, and where the system matrix $A(k)$ is such that it switches between the matrices $A_{i} \in \mathbb{R}^{n \times n}$ belonging to the set $\mathscr{A}=\left\{A_{1}, \ldots, A_{m}\right\}$. We shall refer to this as the switching system. The time-invariant discrete time linear system $x(k+1)=A_{i} x(k)$, denoted $\Sigma_{A_{i}}$ is referred to as the $i^{t h}$ constituent system.

Following [2] we assume that (amongst other conditions) the $A_{i}$ matrices in $\mathscr{A}$ are diagonalizable, and any two of the $A_{i}$ matrices share at least $n-1$ real linearly independent eigenvectors. Before we proceed, we exploit in what follows the known fact that for linear systems, uniform global attractivity of the equilibrium state implies global uniform asymptotic stability [5]. With this in mind, we note that while our main result concerns global attractivity of the origin of (1) under certain conditions, the implication is asymptotic stability.

Theorem 2.1 Let $\mathscr{V}=\left\{v_{1}, \ldots, v_{n+1}\right\}$ be a set of real vectors, where each $v_{i} \in \mathbb{R}^{n}$ for $i=\{1,2, \ldots, n+1\}$. Suppose any choice of $n$ vectors in $\mathscr{V}$ are linearly independent. For each $i \in\{1,2, \ldots, n+1\}$, we construct $M_{i} \in \mathbb{R}^{n \times n}$ matrices as follows

$$
M_{i}=\left\{\begin{array}{l}
{\left[v_{1}, v_{2}, \ldots, v_{n-1}, v_{n}\right] \text { for } \quad i=1} \\
{\left[v_{1}, \ldots, v_{n+1}, v_{i}, \ldots, v_{n}\right] \text { for } 2 \leq i \leq n+1}
\end{array}\right.
$$

i.e., for $i \neq 1, M_{i}$ is obtained by replacing the $(i-1)^{\text {th }}$ column in $M_{1}$ with the vector $v_{n+1}$. Suppose we also have $p$ different diagonal matrices $D_{1}, D_{2}, \ldots, D_{p}$ in $\mathbb{R}^{n \times n}$ with all diagonal entries in $(0,1)$. We now define the matrices $A_{h, i} \in \mathbb{R}^{n \times n}$ as follows

$$
A_{h, i}=M_{i} D_{h} M_{i}^{-1},
$$

and let $\mathscr{A}$ be the set of all $A_{h, i}$ for $h \in\{1,2, \ldots, p\}$ and $i \in\{1,2, \ldots, n+1\}$. Then for the switching system (1) with the set $\mathscr{A}$ defined as above, the origin is globally attractive.

Comment 2.1 Before we give the proof, we wish to make the following comment. The proof of this theorem is analogous to the proof of Theorem 3.1 in [2]. The main differences in the two proofs are that here we look at the discrete-time case (as opposed to the continuous-time), and significantly that this discrete time proof only works if the eigenvalues of the matrices in $\mathscr{A}$ are in $(0,1)$ (as opposed to $(-1,1)$ ).

Comment 2.2 The proof of Theorem 2.1 given below hinges on equation (17). We need the right hand side of (17) to not change sign as the discrete time step $k$ stays in any switching interval (as was the case in equation (24) in [2]). This is guaranteed by the assumption that the eigenvalues of the matrices in $\mathscr{A}$ are in $(0,1)$. Example 1.1 above shows that Theorem 2.1 is not valid if there is no restriction on the eigenvalues in $(-1,1)$. We will now give the details of the proof up to equation (17), which is the discrete time analogue of equation (24) in [2], and the proof thereafter follows as in [2].

\section{Proof of Theorem 2.1:}

Step-1 : In this step we replace the $n \times n$ matrices $M_{j}$ and

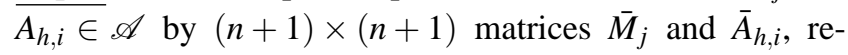
spectively. The matrices $\bar{A}_{h, i} \in \overline{\mathscr{A}} \triangleq\left\{\bar{A}_{h, i}: A_{h, i} \in \mathscr{A}\right\}$ are chosen such that there is at least one common eigenvector $\tau=\left(\begin{array}{llll}1 & 0 & \ldots & 0\end{array}\right)^{T}$ for all the matrices in $\overline{\mathscr{A}}$. The fol- 
lowing lemma helps us construct the augmented matrices $\bar{M}_{j} \in \mathbb{R}^{(n+1) \times(n+1)}$ in the higher dimensional state space.

Lemma 2.1 [2]: Let $\mathscr{V}=\left\{v_{1}, \ldots, v_{n+1}\right\}$ be a set of real vectors with each $v_{i} \in \mathbb{R}^{n}$ for $i=\{1,2, \ldots, n+1\}$. Suppose any choice of $n$ vectors in $\mathscr{V}$ are linearly independent. Then there exists a positive number " $a$ " such that the set $W=$ $\left\{\left(a, v_{1}\right),\left(1, v_{2}\right),\left(1, v_{3}\right), \ldots,\left(1, v_{n+1}\right)\right\}$ is linearly independent in $\mathbb{R}^{n+1}$. Here $\left(a, v_{1}\right)$ is the vector with $n+1$ coordinates, whose first coordinate is " $a$ " and remaining $n$ coordinates are the $n$ coordinates of $v_{1}$.

Based on this lemma we now define matrices $\bar{M}_{i} \in$ $\mathbb{R}^{(n+1) \times(n+1)}$ with a special structure such that they embed the $M_{i} \in \mathbb{R}^{n \times n}$ matrices defined in (2) as follows

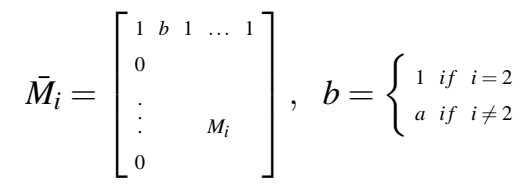

where $i \in\{1,2, \ldots, n+1\}$, and " $a$ " is a scalar as defined in Lemma 2.1. This structure for $\bar{M}_{i}$ was used to ensure that its columns are linearly independent for each $i$. We define matrices $\bar{D}_{h} \triangleq \operatorname{diag}\left(0, D_{h}\right) \in \mathbb{R}^{(n+1) \times(n+1)}$. We now define the matrices $\bar{A}_{h, i}$ and the set $\overline{\mathscr{A}}$ as follows

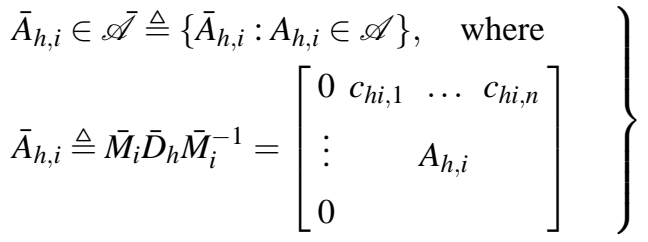

for some real numbers $c_{h i, 1}, c_{h i, 2}, \ldots, c_{h i, n}$ that depend on $h$ and $i$. Note here that $\tau=\left(\begin{array}{llll}1 & 0 & \ldots & 0\end{array}\right)^{T}$ is a common eigenvector for all the $m=p(n+1)$ number of matrices $\bar{A}_{h, i} \in \overline{\mathscr{A}}$. We can now express the $(n+1)^{t h}$ order state space system with the augmented matrices $\bar{A}_{h, i} \in \overline{\mathscr{A}}$ as follows

$$
\left(\begin{array}{c}
x_{n+1}(k+1) \\
x_{1}(k+1) \\
\vdots \\
x_{n}(k+1)
\end{array}\right)=\bar{A}_{h, i}\left(\begin{array}{c}
x_{n+1}(k) \\
x_{1}(k) \\
\vdots \\
x_{n}(k)
\end{array}\right)
$$

which according to the special structure assumed for $\bar{A}_{h, i}$ in (5), is valid if and only if the following set of equations hold

$$
\left.\begin{array}{rl}
\left(\begin{array}{c}
x_{1}(k+1) \\
\vdots \\
x_{n}(k+1)
\end{array}\right) & =A_{h, i}\left(\begin{array}{c}
x_{1}(k) \\
\vdots \\
x_{n}(k)
\end{array}\right) \\
x_{n+1}(k+1) & =\sum_{j=1}^{n} c_{h i, j} x_{j}(k)
\end{array}\right\}
$$

It is apparent from this last equation that the higher dimensional switching system with $n+1$ states explicitly contains the original switching system with $n$ states. We will show in step 3 of the proof that for any solution $\bar{x}(k)=$ $\left(x_{n+1}(k), x_{1}(k), x_{2}(k), \ldots, x_{n}(k)\right)$ of the augmented switching system (6), $\lim _{k \rightarrow \infty}\left(x_{1}(k), x_{2}(k), \ldots, x_{n}(k)\right)=0$ will be guaranteed for any solution $x(k)=\left(x_{1}(k), x_{2}(k), \ldots, x_{n}(k)\right)$ of the original switched system (1) with the special structure, thus proving global attractivity of the origin.

Step-2 : Now for a given $i \in\{1,2, \ldots, n+1\}$ we consider $\overline{\text { the } n}+1$ linearly independent columns of $\bar{M}_{i}$. These form an $n+1$ dimensional coordinate system which includes $\tau$ as one of the axes. We consider the projection of the state $\bar{x}(k)$ onto $\tau$ as the dynamics of the system (6) evolve. This projection is given by the first component of the vector

$g_{i}(k)=\bar{M}_{i}^{-1} \bar{x}(k)$

and is denoted by $\left[g_{i}\right]_{1}(k)$. We denote the $j^{t h}$ component of $g_{i}(k)$ as $\left[g_{i}\right]_{j}(k)$ for each $i=\{1,2, \ldots, n+1\}$. We define

$$
G(k)=\left\{\left[g_{1}\right]_{1}(k),\left[g_{2}\right]_{1}(k),\left[g_{3}\right]_{1}(k), \ldots,\left[g_{n+1}\right]_{1}(k)\right\} .
$$

Now suppose that the system dynamics of the augmented system (6) are described by the following LTI system

$$
\bar{x}(k+1)=\bar{A}_{h, j} \bar{x}(k)
$$

during some arbitrary discrete time interval $\left[k_{1}, k_{2}\right]$, where $k_{2}=k_{1}+s$ for some positive integer $s$ representing the number of discrete time steps. Then

$$
g_{j}(k+1)=\bar{D}_{h} g_{j}(k) .
$$

We denote $\lambda_{h, m}$ as the $m^{\text {th }}$ diagonal element of the $n \times$ $n$ diagonal matrices $D_{h}$ for $m \in\{1,2, \ldots, n\}$ and for $h \in$ $\{1,2, \ldots, p\}$. It follows from the definition of $D_{h}$ that $\lambda_{h, m}$ is the $(m+1)^{t h}$ diagonal element of $\bar{D}_{h}$. Suppose further that each eigenvalue $\lambda_{h, m}$ is in $(0,1)$. We then have

$\left[g_{j}\right]_{m}(k+1)=\left\{\begin{array}{cc}0 & \text { for } m=1 \\ \lambda_{h, m-1}\left[g_{j}\right]_{m}(k) & \text { for } m \neq 1\end{array}\right.$.

Thus, when we are system (10) we get

$$
\left[g_{j}\right]_{m}(k)=\left(\lambda_{h, m-1}\right)^{k-k_{1}}\left[g_{j}\right]_{m}\left(k_{1}\right) \quad \text { for } \quad m \neq 1
$$

Note here that $\left[g_{j}\right]_{1}(k)$ is a constant function of the discrete time step $k$, while each $\left[g_{j}\right]_{m}(k)$ for $m \neq 1$ varies according to relationship (13) over the discrete interval $\left[k_{1}, k_{2}\right]$. Consider the evolution of $\left[g_{i}\right]_{1}(k)$ relative to $\left[g_{j}\right]_{1}(k)$. This 'distance' denoted by $d_{i, j}(k)$ is given by

$$
d_{i, j}(k)=\left|\left[g_{i}\right]_{1}(k)-\left[g_{j}\right]_{1}(k)\right| .
$$


Note that using the following identity

$$
g_{i}(k)=\bar{M}_{i}^{-1} \bar{M}_{j} g_{j}(k)
$$

one can conveniently calculate the distance $d_{i, j}(k)$ as the first component of the vector $\left|\left(\bar{M}_{i}^{-1} \bar{M}_{j}-I\right) g_{j}(k)\right|$, where $I$ is the identity matrix in $\mathbb{R}^{(n+1) \times(n+1)}$. Looking at the structure of the matrix $F_{i, j}=\bar{M}_{i}^{-1} \bar{M}_{j}$ for $i \neq j$, we observe that the firstrow first-column entry of this matrix is always 1 . Next, we give a lemma which establishes that there is only one other nonzero entry in the first row of $F_{i, j}$ matrix.

Lemma 2.2 [2] If we exclude the first column of the matrix $F_{i, j}=\bar{M}_{i}^{-1} \bar{M}_{j}$, for $i \neq j$, then there is only one non-zero entry denoted by $c_{i, j, \delta}$ in the first row, and column $\delta$. Note that $\delta$ depends on the $i, j$ indices, and the relationship is given as

$\delta=\left\{\begin{array}{l}j \text { when } i=1 \\ i \text { when } i \neq 1\end{array}\right.$

Using this lemma and the identity (15) it can be shown that

$$
\left[g_{i}\right]_{1}(k)=\left[g_{j}\right]_{1}(k)+c_{i, j, \delta}\left[g_{j}\right]_{\delta}(k),
$$

for $1 \leq i \leq n+1$ and $i \neq j$, which is valid irrespective of the switched system that we are in. Combining (13) and (16) we get

$\left[g_{i}\right]_{1}(k)-\left[g_{j}\right]_{1}(k)=c_{i, j, \delta}\left(\lambda_{h, \delta-1}\right)^{k-k_{1}}\left[g_{j}\right]_{\delta}\left(k_{1}\right)$,

where $i \neq j$ and whenever we are in system (10).

Step-3 : In this last step we show that $\lim _{k \rightarrow \infty} \mid\left[g_{i}\right]_{1}(k)-$ $\overline{\left[g_{j}\right]_{1}(k)} \mid=0$, for all $i, j \in\{1, \ldots, n+1\}$. From this fact we will deduce that $\lim _{k \rightarrow \infty}\left(x_{1}(k), \ldots, x_{n}(k)\right)=0$. Hence, the Theorem will be proved.

The proof of this step follows along the same lines as the proof of Theorem 3.1 in [2]. Note that equation (17) in this paper is the analogue of equation (24) in [2], and the proofs thereafter are analogous. As mentioned in Comment 2.2, the proof from here on works because the eigenvalues $\lambda_{h, \delta-1}$ are in $(0,1)$.

\section{A stabilizing switched controller design for config- urable driving experience for automotive vehicles}

As an example of the application of the results presented in the previous section we consider the design of an automobile roll dynamics enhancement system. Software configurable driving experience enhancement technologies utilizing active control systems is a topical subject for many car manufacturers. In fact, there are already some passenger vehicles

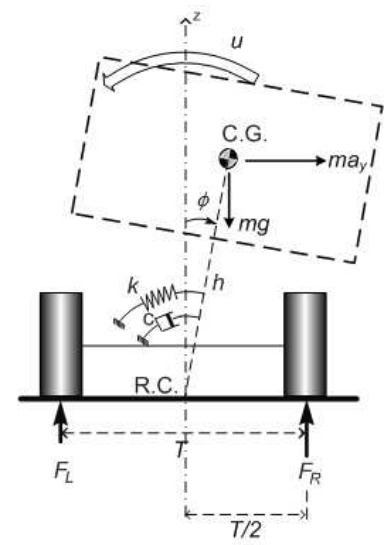

Fig. 1. Second order roll plane model.

on the market that give the drivers the option to select comfort and sporty driving experience settings with a press of a button, and/or modify the suspension settings as a function of speed [6]. The aim of the control design given here is to configure the driving experience based on active suspension actuators alone, and at the same time, guarantee switching stability in the roll dynamics of the vehicle subject to sudden changes in the CG (center of gravity) position.

The equations of motion for the simplified roll plane model of a car with roll torque input, shown in Figure 1, is given as

$J_{x_{e q}} \ddot{\phi}+c \dot{\phi}+k \phi=m h\left(a_{y}+g \phi\right)+u$,

where $u$ denotes the roll torque input provided by active suspension actuators, and $\phi$ is the roll angle. Also $a_{y}$ is the lateral acceleration, and $J_{x_{e q}}=J_{x x}+m h^{2}$ denotes the equivalent roll moment of inertia. Further definitions of the parameters appearing in the model is given in Table 1.

It is important here to note that the changes in the CG position significantly affects the roll dynamics of a vehicle [1]. Given that these changes in the CG position can be detected in real time (for an example of such a method see [7]), we give next a synthesis method for a stable switched linear control design procedure for driving dynamics enhancement system based on active suspension actuators, and making use of the results obtained in Section 2. For illustrative purposes, we assume vertical changes in CG position as the only source of switching, which can result from vertical load shifts and/or passenger movements.

The switched control structure consists of $N$ different controllers that switch based on the current $\mathrm{CG}$ height (i.e., the $\mathrm{CG}$ height change is the switching criteria). For the ease of exposition we assume $N=3$. Defining the state as $x=[\phi, \dot{\phi}]^{T}$, and using a first order approximation for the matrix exponentials, we can represent (18) as in the following discrete time state space form

$x(k+1)=A_{d, i} x(k)+G_{d, i} a_{y}(k)+B_{d, i} u(k)$, 
where $i \in\{1,2,3\}$ and

$$
\left.\begin{array}{rl}
A_{d, i}= & {\left[\begin{array}{cc}
1 & \Delta t \\
-\frac{\left(k-m g h_{i}\right) \Delta t}{J_{x_{e q, i}}} & 1-\frac{c \Delta t}{J_{x_{e q, i}}}
\end{array}\right],} \\
G_{d, i}= & {\left[\begin{array}{ll}
0 & \frac{m h_{i} \Delta t}{J_{x_{e q, i}}}
\end{array}\right]^{T} B_{d, i}=\left[\begin{array}{ll}
0 & \frac{\Delta t}{J_{x_{e q, i}}}
\end{array}\right]^{T}}
\end{array}\right\}
$$

We propose the following switched linear state feedback control structure

$C_{i}: u_{i}(k)=-K_{i} x(k) \quad$ for $\quad i \in\{1,2,3\}$,

where $K_{i}=\left[\kappa_{i 1}, \kappa_{i 2}\right]$ with $\kappa_{i 1}, \kappa_{i 2} \in \mathbb{R}$, are fixed control gains corresponding to each $\mathrm{CG}$ height configuration. Then, the closed loop system can be expressed as $x(k+1)=$ $\tilde{A}_{i} x(k)+G_{d, i} a_{y}(k)$, where

$\tilde{A}_{i}=A_{d, i}-B_{d, i} K_{i}=\left[\begin{array}{cc}1 & \Delta t \\ -\frac{\left(k-m g h_{i}+\kappa_{i 1}\right) \Delta t}{J_{x_{e q, i}}} & 1-\frac{\left(c+\kappa_{i 2}\right) \Delta t}{J_{x_{e q, i}}}\end{array}\right]$

for each $i \in\{1,2,3\}$. The following lemma states the controller synthesis procedure.

Lemma 3.1 [6] Let the matrices $\tilde{A}_{i} \in \mathbb{R}^{2 \times 2}$ for $i \in\{1,2,3\}$ be given as defined in (22). Consider $D_{1}, D_{2}, D_{3} \in \mathbb{R}^{2 \times 2}$

$D_{1}=\left[\begin{array}{cc}\lambda_{1} & 0 \\ 0 & \lambda_{2}\end{array}\right], D_{2}=\left[\begin{array}{cc}\lambda_{3} & 0 \\ 0 & \lambda_{2}\end{array}\right], D_{3}=\left[\begin{array}{cc}\lambda_{1} & 0 \\ 0 & \lambda_{3}\end{array}\right]$,

where the diagonal elements are such that $0<\lambda_{j}<1$ and $\lambda_{i} \neq \lambda_{j}$ for every $i, j \in\{1,2,3\}$ and $i \neq j$. Suppose further that invertible matrices $M_{1}, M_{2}, M_{3} \in \mathbb{R}^{2 \times 2}$ are defined as

$M_{1}=\left[\begin{array}{ll}v_{1} & \mu_{1} \\ v_{2} & \mu_{2}\end{array}\right], M_{2}=\left[\begin{array}{ll}\eta_{1} & \mu_{1} \\ \eta_{2} & \mu_{2}\end{array}\right], M_{3}=\left[\begin{array}{ll}v_{1} & \eta_{1} \\ v_{2} & \eta_{2}\end{array}\right]$

where all the entries $v_{1}, \eta_{1}, \mu_{1}, \nu_{2}, \eta_{2}, \mu_{2}$ are real numbers. Then the following control gains $\kappa_{i 1}, \kappa_{i 2}$ for each $i$

$$
\left.\begin{array}{l}
\kappa_{11}=m g h_{1}-k-\frac{J_{x_{e q, 1}}}{\Delta t^{2}}\left(\lambda_{1}-1\right)\left(\lambda_{2}-1\right) \\
\kappa_{12}=-c+\frac{J_{x_{e q, 1}}}{\Delta t} \frac{\left(\lambda_{1}-1\right)^{2}-\left(\lambda_{2}-1\right)^{2}}{\lambda_{2}-\lambda_{1}}
\end{array}\right\} \text { for } i=1
$$

guarantee that the conditions of Theorem 2.1 are satisfied, and consequently the switching system is stable.
Table 1

Simulation parameters

\begin{tabular}{clll}
\hline Parameter & Description & Value & Unit \\
\hline$m$ & Vehicle mass & 1200 & {$[\mathrm{~kg}]$} \\
$g$ & Gravitational constant & 9.81 & {$\left[\mathrm{~m} / \mathrm{s}^{2}\right]$} \\
$J_{x x}$ & Roll moment of inertia & 300 & {$\left[\mathrm{kgm}^{2}\right]$} \\
$c$ & susp. damping coeff. & 5000 & {$\left[\mathrm{kgm}^{2} / \mathrm{s}\right]$} \\
$k$ & susp. spring stifness & 30000 & {$\left[\mathrm{kgm}^{2} / \mathrm{s}^{2}\right]$} \\
$h_{1}, h_{2}, h_{3}$ & respective CG heights & $0.5,0.7,0.9$ & {$[\mathrm{~m}]$} \\
\hline
\end{tabular}

Example 3.1 Let the positive constants $\lambda_{1}, \lambda_{2}, \lambda_{3}$ be given as $0.994,0.6,0.3$, respectively. Without loss of generality, we choose the constants $v_{2}, \mu_{2}, \eta_{2}$ as $1,2,3$, respectively. Also, we set the discrete time step as $\Delta t=0.05$. The vehicle model parameters used in the example are given in Table 1 , and they correspond to a compact class vehicle. In this example we assume that the CG height of the vehicle can switch between any of the values $h_{1}, h_{2}$ or $h_{3}$ specified in Table 1 at any instant. Now utilizing Lemma 3.1, the closed loop system matrices $\tilde{A}_{1}, \tilde{A}_{2}$ and $\tilde{A}_{3}$ corresponding to the controller gains $\kappa_{i 1}, \kappa_{i 2}$ for each $i \in\{1,2,3\}$ are computed as

$\tilde{A}_{1}=\left[\begin{array}{cc}1 & 0.05 \\ -0.048 & 0.594\end{array}\right], \quad \tilde{A}_{2}=\left[\begin{array}{cc}1 & 0.05 \\ -5.6 & -0.1\end{array}\right], \quad \tilde{A}_{3}=\left[\begin{array}{cc}1 & 0.05 \\ -0.084 & 0.294\end{array}\right]$

Then the evolution of dynamics corresponding to any switching sequence between the unforced closed loop system matrices $\tilde{A}_{1}, \tilde{A}_{2}$ and $\tilde{A}_{3}$ are stable by Theorem 2.1 . That is, the switched unforced discrete time dynamical systems expressed as follows

$x(k+1)=A(k) x(k), \quad A(k) \in\left\{\tilde{A}_{1}, \tilde{A}_{2}, \tilde{A}_{3}\right\}$,

are stable under arbitrary switches, and the resulting system matrices have positive real eigenvalues in $(0,1)$. This inherently implies that with the suggested switched control structure, where controller switching is based on the current CG height, results in stable roll dynamics of the vehicle regardless of the switching parameters. Also, it can be shown that the closed loop forced switched roll plane model given with $x(k+1)=\tilde{A}_{i} x(k)+G_{d, i} a_{y}(k)$ is stable for bounded lateral acceleration $a_{y}(k)$ inputs [6].

Comment 3.1 It is important to note here that the closed loop system matrices $\tilde{A}_{i}$ for $i \in\{1,2,3\}$ given in (28) do not have a CQLF, but nevertheless the corresponding switching system is exponentially stable. The non-existence of a CQLF can be confirmed numerically using LMI solvers.

We finally give the numerical simulation results corresponding to the suggested controller in feedback loop with a simple vehicle model known as the "single track model with the roll degree of freedom" [6]. We used this model to represent the real vehicle in simulation and in a feedback loop with the discrete time control design introduced earlier. The reference maneuver is a steady state cornering maneuver with 

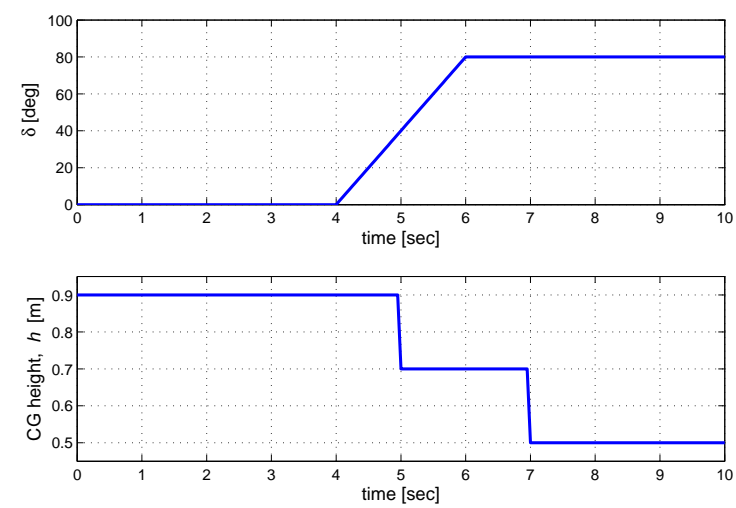

Fig. 2. Driver steering wheel input $\delta$ (where steering ratio is $1 / 20$ ) and the time varying CG height during the maneuver.
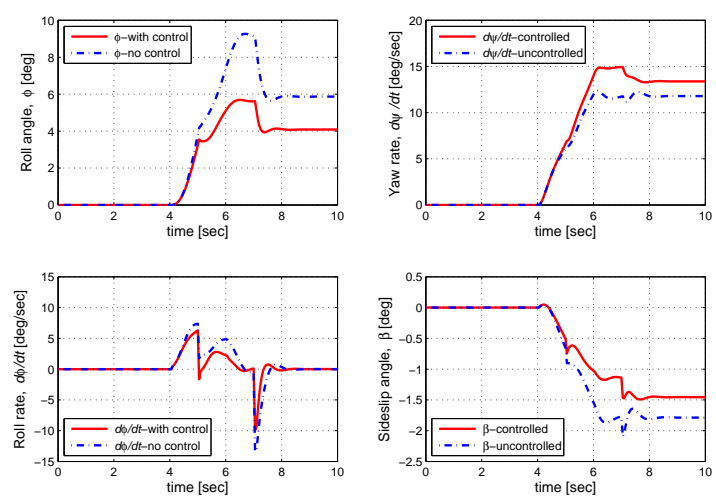

Fig. 3. Comparisons of the states for vehicles with and without control.

a gradual step steering input $(\delta)$ as shown in the upper part of Figure 2. The steering input starts at 4 seconds into the simulation and reaches its peak steady state value of $80^{\circ}$ at 6 seconds in an affine linear fashion. Also the vehicle velocity during the simulation was fixed at $v_{x}=20 \mathrm{~m} / \mathrm{s}$. In order to represent the switching in the dynamics we assumed the CG height profile shown in the lower part of Figure 2, which we assume results from loads falling over inside the vehicle during the maneuver. Using this controller results in the state histories during the maneuver shown in Figure 3 . Note also in the results that the states are compared to those of an uncontrolled vehicle subject to the same parameter switches, and the effectiveness of the controller is evident from the results. Specifically, it is observed from the roll angle and the roll rate profiles (shown in the left half of figure) that the suggested switched controller reduces the controlled roll angle significantly while preserving the vertical response characteristics. This implies that for a given steering input, the controlled vehicle can tolerate higher yaw rates without having as much sideslip, which is desirable in terms of improved cornering performance for a sporty driving experience.

\section{Concluding remarks}

In this paper we have shown that the global attractivity results for a class of discrete-time switching systems is not necessarily equivalent to continuous time systems with this property. Hence, in cases when the existence of a CQLF is unknown for the switched set of LTI systems, qualitative statements concerning the system stability for the continuous-time as well as the discrete-time systems must be validated separately using non-CQLF techniques. One such technique for a specific class of systems is presented in this paper. This result can be translated into practical control design laws for switched systems, which we demonstrated by a controller synthesis procedure for the stabilization of automotive roll dynamics subject to switches in the CG height.

\section{Acknowledgements}

This work was supported by SFI Grant 04/IN3/I478. The authors would to thank and gratefully acknowledge Dr. Oliver Mason as well as all the anonymous reviewers whose many helpful and insightful comments greatly improved both the content and the presentation of the paper.

\section{References}

[1] Solmaz, S., Corless, M., \& Shorten, R. (2007). A methodology for the design of robust rollover prevention controllers for automotive vehicles: Part 2-active steering. Proceedings of the American Control Conference, New York, pp. 1606-1611.

[2] Shorten, R., \& Ó Cairbre, F. (2001). A proof of global attractivity for a class of switching systems using a non-quadratic Lyapunov approach. IMA Journal of Mathematical Control and Information, 18, 341-353.

[3] Wulff, K., Wirth, F., \& Shorten, R., (2005). On the stabilisation of a class of switched single-input single-output systems. IEEE Conference on Decision and Control and European Control Conference, Sevilla.

[4] Mori, T., Nguyen, T.V., Mori, Y., \& Kokame, H. (2006) Preservation of Lyapunov functions under bilinear mapping. Automatica 42, 10551058.

[5] Rugh, W. J. (1996). Linear systems theory, 2nd Ed., New Jersey: Prentice-Hall Information and System Sciences Series.

[6] Solmaz, S. (2007). Topics in automotive rollover prevention: robust and adaptive switching strategies for estimation and control. PhD thesis, Hamilton Institute, National University of Ireland-Maynooth. Available online from the following web link: www.hamilton.ie/publications/PhD_thesis_SelimSolmaz_Dec2007.pdf

[7] Solmaz, S., Akar, M., \& Shorten, R. (2006). Method for determining the center of gravity for an automotive vehicle. Irish Patent Ref: (S2006/0162) 